\title{
AVALIAÇÃO INICIAL DE PROGÊNIES DE MANGABEIRA
}

\author{
CARDOSO, Milena Nascimento ${ }^{1}$ \\ NASCIMENTO, Ana Letícia Sirqueira ${ }^{1}$ \\ LEDO, Ana Silva ${ }^{2}$ \\ SILVA, Ana Veruska Cruz ${ }^{2}$
}

RESUMO: A mangabeira (Hancornia speciosa Gomes) é uma espécie frutífera nativa do Brasil. A avaliação de características de crescimento se torna importante em pesquisas de melhoramento genético e podem estar correlacionadas positivamente com produtividade. O presente trabalho objetivou avaliar aspectos iniciais de desenvolvimento em um teste de progênie, que possibilite fundamentos para conservação da mangabeira. Foram avaliadas as variáveis: altura das mudas, diâmetro do coleto e sobrevivência. O delineamento experimental utilizado foi em blocos casualizados, em que os tratamentos consistiram nos diferentes acessos utilizados na produção das mudas e nos tempos de avaliação. Para comparação do tempo de crescimento foi utilizado o teste de Tukey $(\mathrm{p}<0,5)$, para a influência dos acessos foi utilizado o teste de Skott-Knott $(\mathrm{p}<0,5)$ e para o estudo da sobrevivência foi utilizado o Teste $\mathrm{t}(\mathrm{p}<0,5)$. Constatou-se que diferenças de crescimento e sobrevivência verificadas em campo é um indicativo de que matrizes selecionadas em ambiente natural servem como reservatório de recursos genéticos.

Palavras-chave: Hancornia Speciosa Gomes. Produtividade. Conservação.

\section{INITIAL EVALUATION OF MANGABEIRA PROGENIES}

SUMMARY: Mangaba tree (Hancornia speciosa Gomes) is a fruit species native to Brazil. The evaluation of growth traits become important in genetic improvement research and may be positively correlated with productivity. The aim of the study was to evaluate initial aspects of development in a progeny test, which provides a basis for the conservation of mangaba. The following variables were evaluated: height of seedlings, collar diameter and survival. The experimental design was a randomized complete block design, where the treatments consisted of the different accession used in the production of the seedlings and in the evaluation times. The Tukey test $(p<0,5)$ was used to compare the growth time, the Skott-Knott test $(\mathrm{p}<0,5)$ was used for the influence of the accesses and for the survival study the Test $t(p<0,5)$. It was verified that differences of growth and survival in the field is an indication that matrices selected in natural environment serve as reservoir of genetic resources.

Keywords: Hancornia Speciosa Gomes. Productivity. Conservation.

\section{INTRODUÇÃO}

A mangabeira (Hancornia speciosa Gomes), pertencente à família Apocynaceae, é uma espécie frutífera nativa do Brasil, característica dos Tabuleiros Costeiros e de faixas litorâneas da região Nordeste do país, como também dos Cerrados das regiões Centro-Oeste, Norte e Sudeste (SILVA JUNIOR, 2004; FERREIRA et al., 2013).

Os frutos são altamente apreciados pelos consumidores devido às suas características organolépticas e considerável valor nutricional (SILVA et al., 2017). Podem ser consumidos in natura ou processado na fabricação de sucos, geleias, xarope, licor, doces e sorvetes, e ainda apresentarem características de usos medicinais, utilizadas principalmente pelas comunidades extrativistas (FERREIRA; MARINHO, 2007).

\footnotetext{
${ }^{1}$ UFS

${ }^{2}$ Embrapa Tabuleiros Costeiros
} 
Para a produção de mudas o substrato exerce influência sobre a germinação, uma vez que fatores como estrutura, aeração, capacidade de retenção de água e grau de contaminação por patógenos podem variar segundo o material utilizado, sendo estes decisivos no desenvolvimento e sobrevivência da espécie (NOGUEIRA et al., 2003). Além disso, as sementes são recalcitrantes, não tolerando armazenamento, devendo ser semeadas após a extração (AGUIAR FILHO et al., 1998).

A espécie requer temperaturas entre 24 e $26^{\circ} \mathrm{C}$ e chuvas bem distribuídas, variando de 750 a 1.600 $\mathrm{mm}$ anuais. Desenvolve-se bem em solos arenosos e pobres, embora responda positivamente ao cultivo em solos com maior grau de fertilidade, frutificando principalmente entre os meses de julho a outubro ou de janeiro a abril (LÉDO et al., 2015).

A mangabeira encontra-se em fase de domesticação e necessita de estudos que envolvam os aspectos relacionados ao seu cultivo, seleção de genótipos promissores, desenvolvimento e adaptação de práticas culturais da planta (LÉDO et al., 2007). Informações acerca do seu desenvolvimento são fundamentais na domesticação e incorporação em sistemas produtivos regionais, bem como estratégias eficientes de conservação (COSTA et al., 2011).

A avaliação de características de crescimento é importante em pesquisas de melhoramento genético (ASSIS, 2014) e podem estar correlacionadas positivamente com produtividade, servindo de base para auxiliar na seleção direta ou indireta de progênies promissoras dos diversos acessos (SILVA et al., 2015), uma vez que a mangaba é uma frutífera nativa com potencial econômico.

Considerando a instabilidade da espécie e ameaça de extinção recorrente em que está inserida, objetivou-se avaliar aspectos iniciais de desenvolvimento em um teste de progênie, que possibilite fundamentos para conservação da mangabeira.

\section{MATERIAL E MÉTODO}

As sementes utilizadas para a produção das progênies foram provenientes de diferentes acessos do Banco Ativo de Germoplasma da Mangaba (BAGMangaba) localizado no Campo Experimental da

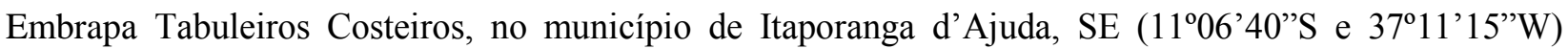
(Tabela 1). Foram coletados frutos caídos e maduros, considerando o formato e coloração dos mesmos, além da carga produtiva das plantas que pertenciam.

Os frutos foram acondicionados em sacos plásticos e transportados até o laboratório de Sementes da Embrapa Tabuleiros Costeiros em Aracaju/SE, onde foi feito o beneficiamento manual com auxílio de peneira. Posteriormente as sementes foram lavadas em água corrente para retirada de mucilagem, para que pudessem ser semeadas. As progênies foram nomeadas de acordo com a codificação do BAG (Tabela 1).

Tabela1. Procedência, codificação e número de plantas utilizados nas progênies derivadas do Banco Ativo de Germoplasma de Mangaba.

\begin{tabular}{ccc}
\hline Procedência / Acesso & Código & $\mathbf{N}^{\mathbf{0}}$ de plantas \\
\hline Jandaíra, BA/ Costa Azul & CAP & 5 \\
\hline Conde, BA/ Barra do Itariri & BIP & 6 \\
\hline Mata de São João, BA/ Lagoa Grande & LGP & 6 \\
\hline Indiaroba, SE/ Terra Caida & TCP & 6 \\
\hline Indiaroba, SE/ Pontal & PTP & 6 \\
\hline Salvaterra, PA/ Água Boa & ABP & 6 \\
\hline
\end{tabular}


A semeadura foi realizada em sacos de polietileno (16x24), contendo substrato tipo areia lavada em número de três sementes por saco. Os sacos permaneceram na casa de vegetação durante o período de 210 dias e posteriormente foram levadas a campo, onde foram transplantadas para uma área típica de restinga, cujo solo é do tipo expodossolo humilúvico durante as avaliações que ocorreram no período de janeiro de 2015 a janeiro de 2017. Foram avaliadas as variáveis descritas a seguir:

Altura das mudas: o desenvolvimento das mudas foi acompanhado anualmente quando transplantadas para o campo. As avaliações foram realizadas com auxílio de régua graduada em centímetros, medindo a partir do solo onde foi emitido o caule até a inserção da última folha e o resultado dividido pelo número de mudas.

Diâmetro do coleto: foi avaliado nos intervalos anteriormente mencionados com auxílio de paquímetro digital.

Sobrevivência: os indivíduos de cada acesso foram contabilizados durante as avaliações anuais

$\mathrm{O}$ delineamento experimental utilizado foi em blocos casualizados, em que os tratamentos consistiram nos diferentes acessos (seis - CAP, BIP, LGP, TCP, PTP, ABP) utilizados na produção das mudas e nos tempos de avaliação (três anos - 2015, 2016 e 2017). Para comparação do tempo de crescimento foi utilizado o teste de Tukey $(\mathrm{p}<0,5)$, para a influência dos acessos foi utilizado o teste de Skott-Knott $(\mathrm{p}<0,5)$ e para o estudo da sobrevivência foi utilizado o Teste $t(\mathrm{p}<0,5)$.

\section{RESULTADO E DISCUSSÃO}

Houve diferença significativa entre os anos e os acessos das progênies de mangabeira para a variável altura, e interação significativa entre as fontes de variação para o diâmetro do coleto (Tabela 2).

A média geral para altura no último ano de avaliação foi 52,31 cm, e os acessos TCP6 $(54,47 \mathrm{~cm})$ e PTP5 $(59,55 \mathrm{~cm})$ apresentaram as maiores médias ao decorrer de todas as avaliações. Rodrigues et al. (2017) avaliando comportamento morfológico no período de crescimento de progênies de cupuaçuzeiros [Theobroma grandiflorum (Willd. ex Spreng.) Schum.] em 180 dias, obtiveram altura média de 9,22 cm, o que demonstra crescimento inferior às progênies de mangabeira.

Sano et al. (2012) ao avaliar progênies de mangabeira, observaram que a progênie de maior crescimento inicial na fase de viveiro foi também a que na fase adulta apresentou plantas com maior produção de frutos, maior altura e maior diâmetro do caule. Tais características podem estar correlacionadas positivamente com produtividade e podem servir de base para auxiliar na seleção direta ou indireta de progênies promissoras dos diversos acessos.

Quanto ao diâmetro do coleto, as progênies apresentaram amplitude de variação de 4,73 $\mathrm{mm}$ a 7,82 mm. Não houve diferença estatística entre os acessos nos anos de 2015 e 2016. Em 2017, os acessos BIP2 e TCP6 tiveram as maiores médias $(7,18$ e 17,95mm respectivamente) Esses resultados foram inferiores ao encontrado por Ganga et al. (2009), em avaliação de parâmetros genéticos em progênies de mangabeira, com diâmetro médio de 33,90 mm, num período de aproximadamente 20 meses em campo, e Martins-Coder e Saldanha (2006), em estudo de progênies de palmiteiro (Euterpe edulis MART) 210 dias após a semeadura. Em testes de progênies, o diâmetro do coleto é uma característica adequada para indicar a diferenciação entre genótipos (NODARI; FANTINI, 2000). 
Tabela 2. Altura e diâmetro de caule de progênies derivadas do Banco Ativo de Germoplasma de Mangaba.

\begin{tabular}{|c|c|c|c|c|c|c|c|c|c|c|c|c|c|}
\hline \multirow{3}{*}{ Anos } & \multicolumn{13}{|c|}{ Acessos } \\
\hline & \multicolumn{13}{|c|}{ Altura (cm) } \\
\hline & CAP5 & ABP2 & PTP2 & LGP3 & BIP1 & TCP2 & PTP4 & ABP1 & BIP2 & PRP5 & TCP6 & PTP5 & Médias \\
\hline 2015 & 35,93 & 43,85 & 38,07 & 34,20 & 41,62 & 38,98 & 38,42 & 45,15 & 41,50 & 49,92 & 39,87 & 47,39 & $41,24 \mathrm{~b}$ \\
\hline 2016 & 48,87 & 38,62 & 50,00 & 47,32 & 50,56 & 47,14 & 51,55 & 49,94 & 49,38 & 51,31 & 56,58 & 63,87 & $50,28 a$ \\
\hline 2017 & 41,66 & 48,00 & 40,00 & 49,50 & 46,00 & 52,51 & 55,50 & 47,00 & 51,00 & 55,00 & 80,50 & 70,00 & $52,31 \mathrm{a}$ \\
\hline Médias & $42,20 \mathrm{~B}$ & $42,59 \mathrm{~B}$ & $43,23 \mathrm{~B}$ & $43,67 \mathrm{~B}$ & $46,06 \mathrm{~B}$ & $46,21 \mathrm{~B}$ & $46,59 \mathrm{~B}$ & $47,14 \mathrm{~B}$ & $47,29 \mathrm{~B}$ & $51,59 \mathrm{~B}$ & $54,47 \mathrm{~A}$ & $59,55 \mathrm{~A}$ & \\
\hline CV $(\%)$ & 24,16 & & & & & & & & & & & & \\
\hline \multicolumn{14}{|c|}{ Diâmetro do coleto (mm) } \\
\hline 2015 & $4,15 \mathrm{Ab}$ & $3,82 \mathrm{Ab}$ & $3,12 \mathrm{Ab}$ & $4,49 \mathrm{Ab}$ & $4,76 \mathrm{Ab}$ & $4,56 \mathrm{Ab}$ & $4,37 \mathrm{Ab}$ & $4,89 \mathrm{Ab}$ & $3,95 \mathrm{Aa}$ & $4,26 \mathrm{Ab}$ & $4,45 \mathrm{Ab}$ & $6,18 \mathrm{Ab}$ & 4,46 \\
\hline 2016 & 4,67 Ab & $4,46 \mathrm{Ab}$ & 5,58 Aab & $4,47 \mathrm{Ab}$ & $4,32 \mathrm{Ab}$ & $4,79 \mathrm{Ab}$ & $5,10 \mathrm{Ab}$ & $4,59 \mathrm{Ab}$ & $5,16 \mathrm{Aa}$ & $5,27 \mathrm{Ab}$ & $6,66 \mathrm{Ab}$ & $7,42 \mathrm{Ab}$ & 4,98 \\
\hline 2017 & $8,12 \mathrm{Ba}$ & $9,35 \mathrm{Ba}$ & 7,38 Ba & $7,11 \mathrm{Ba}$ & $9,41 \mathrm{Ba}$ & $9,34 \mathrm{Ba}$ & $10,73 \mathrm{Ba}$ & $10,68 \mathrm{Ba}$ & 7,18 Aa & $8,81 \mathrm{Ba}$ & $17,95 \mathrm{Aa}$ & $12,18 \mathrm{Ba}$ & 9,58 \\
\hline Médias & 5,04 & 4,73 & 4,43 & 4,85 & 4,82 & 5,65 & 5,32 & 5,54 & 4,57 & 5,21 & 7,17 & 7,82 & \\
\hline $\operatorname{CV}(\%)$ & 15,73 & & & & & & & & & & & & \\
\hline
\end{tabular}

Médias seguidas de letras iguais, minúsculas nas colunas não diferem estatisticamente pelo Teste de Tukey e as maiúsculas nas linhas, pertencem ao mesmo grupo pelo Teste de Skott-Knott, ambos, a 5\% de probabilidade de erro. 
A diminuição da sobrevivência entre os anos de avaliação foi estatisticamente significativa (Tabela 3). Os anos de 2016 e 2017 apresentaram menores médias, indicando grande influência de fatores antrópicos.

A sobrevivência foi baixa em todos os anos avaliados, com mortalidade máxima de $92,30 \%$ para o acesso BIP2 (Figura 1) indicando baixa adaptação das progênies ao local de ensaio. Os acessos com maiores ajustamentos foram TCP2, PTP5 e TCP6 com 45\%, 27,2\% e 25\% de sobrevivência respectivamente, indicando acessos promissores para exploração. Em estudos de Martins-Corder e Saldanha (2006) as progênies de palmiteiro apresentaram maior variabilidade para a porcentagem de sobrevivência com amplitude de 26 a $58 \%$ aos 210 dias.

Figura 1. Porcentagens de sobrevivência dos acessos de mangabeira derivados do Banco Ativo de Germoplasma após três anos de avaliação.

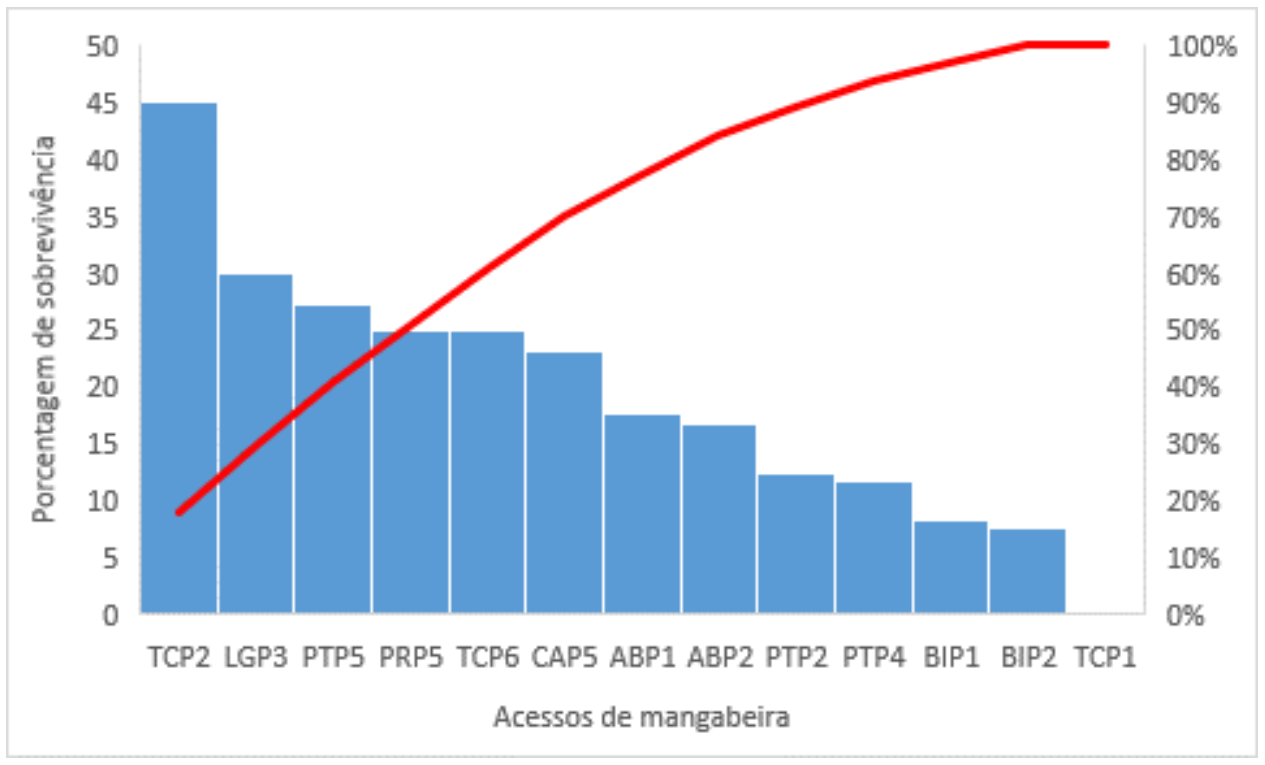

Tabela 3. Sobrevivência de progênies de mangabeira derivadas do Banco Ativo de Germoplasma de Mangaba durante os anos de avaliação.

\begin{tabular}{cccc}
\hline Variáveis de interesse & Média & Teste t & P \\
\hline Sobrevivência & & & $0,0001305^{*}$ \\
\hline 2015 & 4,235 & 5,0013 & $0,0000183^{*}$ \\
\hline 2016 & 7,588 & 6,0065 & $0,0004464^{*}$ \\
2017 & 3,352 & 4,4009 &
\end{tabular}

*diferença estatisticamente ao nível de significância de 5\%.

Resultados de sobrevivência e variáveis biométricas para progênies de Myracrodruon urundeuva com aproximadamente 16 anos, validam a proposta de melhoramento da espécie e as informações levantadas podem servir como base para escolha de acessos com finalidade de recomposição de reserva legal (BERTONHA et al., 2016), demonstrando a importância de avaliações de crescimento de progênies. 


\section{CONCLUSÃO}

A variabilidade existente nas progênies de mangabeira possibilita a seleção e introdução de germoplasmas com características fundamentais ao cultivo. As diferenças de crescimento e sobrevivência verificadas em campo é um indicativo de que matrizes selecionadas em ambiente natural servem como reservatório de recursos genéticos, podendo citar os acessos TCP5 e PTP5.

\section{REFERÊNCIAS}

AGUIAR FILHO, S. P.; BOSCO, J.; ARAÚJO, I. A. de. A mangabeira (Hancornia speciosa): Domesticação e técnicas de cultivo. João Pessoa: EMEPA-PB, 1998, 26p. (EMEPA-PB, Documentos, 24).

ASSIS, G. A.et al.Correlação entre crescimento e produtividade do cafeeiro em função do regime hídrico e densidade de plantio. Bioscience Journal, v.30, n.3, p.666-676, 2014.

BERTONHA, L.J.et al. Seleção de progênies de Myracrodruon urundeuva baseada em caracteres fenológicos e de crescimento para reconstituição de áreas de Reserva Legal. Scientia Forestalis, v. 44, n. 109, p. 95-104, 2016.

COSTA, T. S.et al. Diversidade genética de acessos do banco de germoplasma de mangaba em Sergipe. Pesquisa Agropecuária Brasileira, v.46, p.499-508, 2011.

FERREIRA, E. G.et al. Caracterização molecular de mangabeira (Hancornia speciosa) dos tabuleiros costeiros de Pernambuco e Rio Grande do Norte no Nordeste do Brasil. Revista Verde de Agroecologia e Desenvolvimento Sustentável, v.8, p.7-10, 2013.

FERREIRA, E. G.; MARINHO, S. J. O. Produção de frutos da mangabeira para consumo in natura e industrializado. Tecnologia e Ciência. Agropecuária, v.1, p.9-14, 2007.

GANGA, R. M. D.; CHAVES, L. J.; NAVES, R. V. Parâmetros genéticos em progênies de Hancornia speciosa Gomes do Cerrado. Scientia Florestalis, v.37, n.84, p.395-404, 2009.

LÉDO, A. S.et al. Crescimento inicial de mangabeira (Hancornia speciosa Gomez) em diferentes meios de germinação in vitro. Ciência Agrotecnologia. v. 31, n. 4, p. 115 -125, 2007.

LEDO, A. S.et al. A cultura da mangaba - Brasília, DF: EMBRAPA. 2015, 84p.

MARTINS-CORDER, M. P.; SALDANHA, C. W. Geminação de sementes e crescimento de plântulas de diferentes progênies de Euterpe edulis MART. Revista Árvore, v. 30, n.5, p.693-699, 2006.

NODARI, R. O.; FANTINI, A. C. Melhoramento genético do palmiteiro. Sellowia, v.49-52, p.163-188, 2000.

NOGUEIRA, R. J. M. C.; ALBUQUERQUE, M. B.; SILVA JUNIOR, J. F. Efeito do substrato na emergência, crescimento e comportamento estomático em plântulas de mangabeira. Revista Brasileira de Fruticultura, 2003.

RODRIGUES, J. D. B.et al. Comportamento morfológico no período de crescimento de progênies de cupuaçuzeiro em resposta a restrição hídrica. Ciência \& Tecnologia, v.9, 2017.

SANO, S.M.et al. Avaliação de Progênies de Mangabeira, Espécie Nativa do Cerrado. Boletim de Pesquisa e Desenvolvimento 307. Embrapa Cerrados. 2012. 21p. 
SILVA JUNIOR, J. F. A cultura da mangaba. Revista Brasileira de Fruticultura, Jaboticabal, v.26, p.1$192,2004$.

SILVA, A. V. C.et al. Genetic Diversity of Remaining Populations of Mangaba (Hancornia speciosa Gomes) in Restingas of Brazil. Journal of Agricultural Science, v.9, n.2, 2017.

SILVA, J. A. D.et al. Caracteres de crescimento de progênies de cafeeiros Big Coffee VL. In: SIMPÓSIO DE PESQUISA DOS CAFÉS DO BRASIL, 11. 2015. [Anais...]. Disponível em:

http://www.sbicafe.ufv.br/handle/123456789/4152 